\title{
Theranostics in Metastatic Castrate Resistant Prostate Cancer
}

\author{
Mariza Vorster • Machaba Michael Sathekge \\ Department of Nuclear Medicine, University of Pretoria/Steve Biko Academic Hospital, \\ Pretoria, South Africa
}

Author for correspondence: Mariza Vorster, Department of Nuclear Medicine, University of Pretoria/Steve Biko Academic Hospital, Pretoria, South Africa.

Email: marizavorster@gmail.com

Doi: https://doi.org/10.36255/exonpublications.prostatecancer.theranostics.2021

\begin{abstract}
Despite advances in the treatment of localized prostate cancer, many patients progress to metastatic castration-resistant prostate cancer with limited median survival benefits, and significant morbidity. Therefore, efforts to explore new therapeutic modalities for metastatic castration-resistant prostate cancer are urgently needed. A theranostic approach in oncology is based on the principle of imaging a particular molecular target with a diagnostic radioisotope, and then substituting it with a therapeutic isotope to treat a patient who demonstrates sufficient target expression on diagnostic images. Radioisotope pairs are usually chosen in such a way that their physical and chemical properties are similar to ensure the therapeutic agent will be distributed in the same way as the diagnostic agent. This chapter outlines the most recent advances in the use of prostate specific membrane antigen (PSMA) in theranostics with emphasis on ${ }^{177}$ Lu-PSMA, ${ }^{225} \mathrm{Ac}-\mathrm{PSMA}$ and ${ }^{223} \mathrm{Ra}$-dicloride. The clinical utility of these radioisotopes along with their limitations and future perspectives are discussed.
\end{abstract}

Keywords: ${ }^{225}$ Ac-PSMA; ${ }^{177}$ Lu-PSMA; alpha particle therapy; radioligand therapy; theranostics

In: Prostate Cancer. Bott SRJ, Ng KL (Editors). Exon Publications, Brisbane, Australia. ISBN: 978-0-6450017-5-4; Doi: https://doi.org/10.36255/exonpublications.prostatecancer.2021

Copyright: The Authors.

License: This open access article is licenced under Creative Commons Attribution-NonCommercial 4.0 International (CC BY-NC 4.0) https://creativecommons.org/licenses/by-nc/4.0/ 


\section{INTRODUCTION}

Prostate cancer remains an important cause of morbidity and mortality in men (1) with recent global patterns identifying South Africa as one of the countries with the highest mortality (2). The mainstay of localized cancer treatment consists of prostatectomy, active surveillance and/or radiation therapy, whereas metastatic disease is treated with androgen ablation with or without additional agents, such as docetaxel, abiraterone, enzalutamide, cabazitaxel, and sipuleucel-T (Sip-T) (3). Despite the aforementioned therapies, the majority of patients ultimately progress to metastatic castration-resistant prostate cancer with limited median survival benefits and significant morbidity. The median overall survival for metastatic castration-resistant prostate cancer patients ranges from 13-32 months with a 15\% 5-year survival rate. Therefore, efforts to explore new therapeutic modalities for metastatic castration-resistant prostate cancer are urgently needed (4).

\section{CONVENTIONAL THERAPIES}

Conventional therapy for metastatic prostate cancer consists of androgen deprivation therapy combined with abiraterone acetate plus prednisone, docetaxel, or enzalutamide (4). In the South African setting, initial treatment most commonly includes docetaxel. A recent publication by Abdel-Rahman (5) combined the pooled analysis from three prospective studies which included a total of 1212 patients with metastatic prostate cancer that were treated with the combination of docetaxel and prednisone. Findings indicated a high prevalence of high-grade toxicities, especially neutropenia in older patients. These findings were in line with a number of prior retrospective studies showing a higher risk of treatment toxicities among elderly patients receiving docetaxel for metastatic castrationresistant prostate cancer (5).

\section{THE THERANOSTIC APPROACH}

The theranostic approach in oncology is based on the principle of imaging a particular molecular target with a diagnostic radioisotope, and then substituting it with a therapeutic isotope to treat a patient who demonstrates sufficient target expression on the diagnostic images. Radioisotope pairs are usually chosen in such a way that their physical and chemical properties are similar to ensure that the therapeutic agent will be distributed in the same way as the diagnostic agent (Figure 1). Probably the earliest example of this concept is the use of radioactive iodine to image and treat thyroid cancer, where imaging is performed with ${ }^{123} \mathrm{I}$ and therapy with ${ }^{131} \mathrm{I}$. Another example of such a theranostic pair is ${ }^{68} \mathrm{Ga}$-PSMA (Gallium-68 prostate specific membrane antigen) and ${ }^{177} \mathrm{Lu}-\mathrm{PSMA}$ (Lutetium-177 prostate specific membrane antigen). The radioactive isotope and the target molecule are connected via a ligand or a chelator (6-8). 


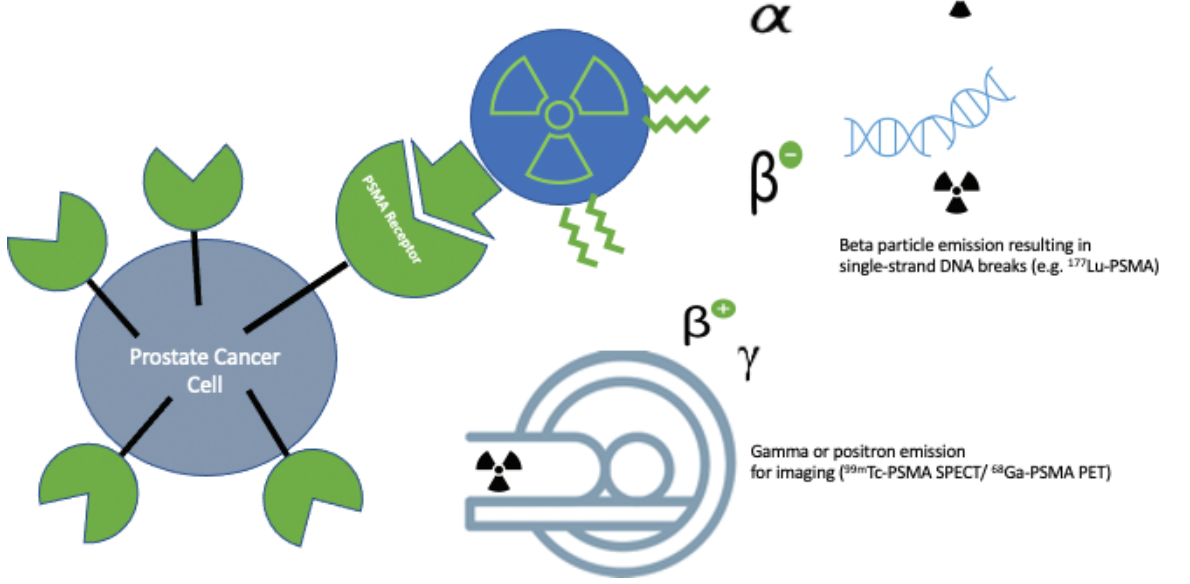

Alpha partide emission resulting in double-stranded DNA breaks (e.g. ${ }^{225}$ Ac-PSMA)

Figure 1. PSMA receptor targeting. Diagrammatic representation of PSMA receptor targeting for cancer detection and / or treatment depending on the radionuclide selection. A theranostic approach consists of first imaging disease presence and extent with a diagnostic isotope prior to targeting disease with a therapeutic isotope.

\section{${ }^{68}$ GA-PSMA}

The superiority of ${ }^{68} \mathrm{Ga}$-PSMA as an imaging modality in prostate cancer management (for example, bone scintigraphy) has been sufficiently demonstrated in multiple comparative studies as well as in a few systematic reviews and meta-analysis (9-11). A 2020 systematic review and meta-analysis published in European Urology concludes that "Ga-68-PSMA positron emission tomography (PET) improves detection of metastases with biochemical recurrence, particularly at low pre-PET prostate-specific antigen (PSA) levels of $>0.2 \mathrm{ng} / \mathrm{ml}(33 \%)$ and $0.2-0.5 \mathrm{ng} / \mathrm{ml}$ (45\%)". A prospective, multi-center study by Hofman et al. (the "proPSMA" study) also convincingly demonstrated the superiority of PSMA-based imaging over computed tomography (CT) and bone scintigraphy (9). Imaging with ${ }^{68} \mathrm{Ga}-\mathrm{PSMA}$ has subsequently been included in the guidelines of the European Association of Urology and National Comprehensive Cancer Network (12). When a theranostic approach is planned, any PSMA-based imaging, labelled to Technetium-99 $\left({ }^{99 \mathrm{~m} T c)}\right.$ or Fluorine-18 $\left({ }^{18} \mathrm{~F}\right)$ may be used for selection and follow-up of patients. However, ${ }^{68} \mathrm{Ga}-\mathrm{PSMA}$ has been best validated to date in this setting.

\section{${ }^{18} \mathrm{~F}-\mathrm{FDG}$}

The use of ${ }^{18}$ F-FDG (Fluorine-18 Fluorodeoxyglucose) seems valuable in screening patients appropriately for targeted radionuclide therapy, although routine 
baseline imaging with both FDG and PSMA may not be practical or cost-effective. Imaging with ${ }^{18} \mathrm{~F}$-FDG PET may have to be reserved for cases with poor clinical and/or biochemical responses despite stable disease, or partial treatment response noted on ${ }^{68} \mathrm{Ga}$-PSMA. This clinical setting may be indicative of prostate cancer that is no longer expressing PSMA in certain metastatic lesions and incongruent uptake of FDG is generally indicative of a poor prognosis (13-15).

\section{${ }^{177}$ LU-PSMA}

PSMA is a Type II transmembrane glycoprotein, consisting of 750 amino acids that is over-expressed in the vast majority of prostate cancer cells. The PSMA receptor has an internalization process that allows endocytosis of bound proteins on the cell surface into an endosomal compartment, which allows PSMA labelled radioisotopes to be concentrated within the cell. The density of expression of this transmembrane receptor on prostate cancer cells further increases depending on the Gleason score of the prostate cancer, and in castrate- resistant prostate cancers, which makes it an ideal target for radionuclide therapy $(16,17)$. A small molecule that specifically binds to the PSMA is commercially available as PSMA-11 and PSMA-617. This molecule consists of a glutamate-urea-lysine that has a high affinity and specificity towards PSMA, a chelator (DOTA in case of PSMA-617 for therapy; N,N'-bis [2-hydroxy-5-(carboxyethyl)benzyl] ethylenediamine-N,N'diacetic acid (HBED-cc) in case of PSMA-11 for diagnosis), and a linker that differs depending on the chelating agent. The linker can be re-designed for optimization of hydrophilicity and biodistribution. PSMA-617 is a theranostic probe that can be used for both imaging and therapy just by changing the radiometal. However, in clinical practice, PSMA-11 is often used for diagnostic staging and the DOTA-analogue PSMA-617 is used for therapy as the biodistribution of the different PSMA ligands is more suitable for each application (18-20).

${ }^{177} \mathrm{Lu}$ is a therapeutic isotope which results in the emission of beta minus particles $\left(\beta^{-}\right)$that have a range between 1 and $10 \mathrm{~mm}$ with energies between 0.1 and $1 \mathrm{MeV}$. This results in multiple single strand breaks in the DNA of the targeted cancer cells. A recent review by Jones et al. (21) on PSMA theranostics summarized current ongoing trials with ${ }^{177} \mathrm{LuPSMA}$. In a more recent review, Emmet (22), highlighted the expanding role of PSMA-directed theranostics in prostate cancer as a sensitive diagnostic tool that can be coupled with efficacious and lowtoxicity therapeutic options.

\section{Mode of administration of ${ }^{177}$ Lu-PSMA}

Patients are selected based on the expression of the PSMA target after PSMAbased imaging, for example, ${ }^{68} \mathrm{Ga}$-PSMA PET/CT or ${ }^{99 \mathrm{~m}} \mathrm{Tc}-\mathrm{PSMA}$ or ${ }^{18} \mathrm{~F}-\mathrm{PSMA}$ (Figure 2). Absence of any contra-indications, such as bone marrow suppression or renal impairment, is also evaluated $(23,24)$. Currently, ${ }^{177} \mathrm{Lu}$-PSMA radioligand therapy (RLT) is administered on a compassionate basis when all traditional therapies have been exhausted. Its exact sequence in the treatment pathway among well-established therapies is uncertain. The guidelines by the European 


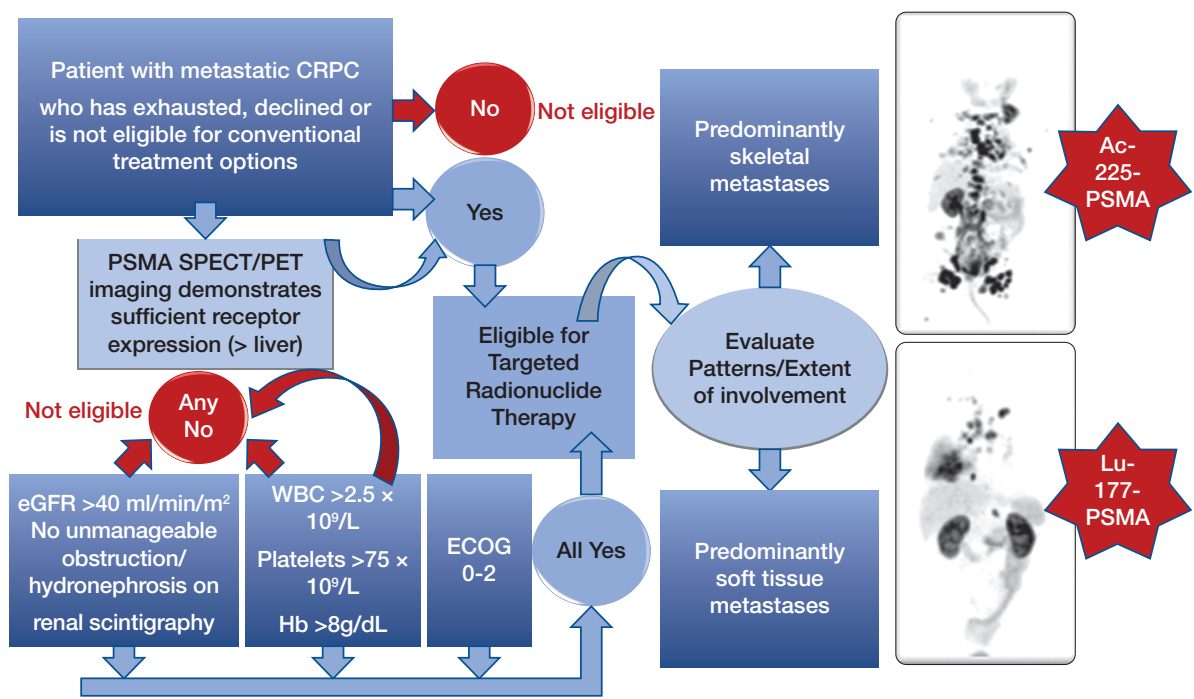

Figure 2. Patient selection for targeted radionuclide therapy. Eligibility evaluation for PSMA radioligand therapy includes demonstrating the presence of sufficient PSMA overexpression on imaging as well as ensuring adequate renal function, bone marrow reserves and ECOG of less than 2. Diffuse skeletal metastases are better suited to treatment with ${ }^{225} \mathrm{Ac}$-PSMA as opposed to the presence of predominantly soft tissue metastases that is better suited to treatment with ${ }^{177} \mathrm{Lu}$-PSMA.

Association of Nuclear Medicine (EANM) state that ${ }^{177}$ Lu-PSMA should be administered in the following way (25): (i) in patients with low cardiovascular risk, 1-2 L normal saline may be given intravenously at $20 \mathrm{cc} / \mathrm{min}$ flow rate; and (ii) RLT is administered by slow intravenous injection of ${ }^{177} \mathrm{Lu}-\mathrm{PSMA}$. The following patient-specific recommendations for RLT can be considered:

(i) Diuretics and moderate laxatives can be given after RLT to support clearance of unbound ${ }^{177}$ Lu-PSMA.

(ii) Cold packs applied to salivary glands could eventually reduce ${ }^{177}$ Lu-PSMA uptake during the blood pool phase. The value of cold packs is still controversial.

(iii) Prophylactic antiemetic therapy, for example, ondansetron.

(iv) Corticosteroids one day before, and up to several days after RLT are mandatory in case of cerebral, spinal or other metastases with risk of painful or obstructive swelling; otherwise, they are optional and case-dependent.

These guidelines have also been adapted to the South African context and published (26).

\section{Efficacy, safety and outcomes of ${ }^{177}$ Lu-PSMA}

Several retrospective studies performed worldwide have demonstrated promising improvements in progression-free and overall survival (27-30). Hofman et al. (24) 
performed the first large prospective study at the Peter MacCallum Cancer Centre in Melbourne, Australia. The researchers recruited men with metastatic castrationresistant prostate cancer and progressive disease after standard treatments, including taxane-based chemotherapy and second-generation anti-androgens. Eligible patients had progressive disease as defined by imaging (according to Response Evaluation Criteria In Solid Tumors [RECIST] or bone scan) or new pain in an area of radiographically evident disease, and were required to have an Eastern Cooperative Oncology Group (ECOG) performance status score of 2 or lower. The study participants received up to four cycles of intravenous ${ }^{177} \mathrm{Lu}-\mathrm{PSMA}-617$ with a mean dose of $7.5 \mathrm{GBq} / \mathrm{cycle}$, at six weekly intervals. Primary endpoints consisted of a PSA response according to Prostate Cancer Clinical Trial Working Group criteria (defined as a greater than 50\% PSA decline from baseline) and imaging responses (as measured by bone scan, CT, PSMA, and FDG PET/CT) and quality of life (assessed with the EORTC-Q30 and Brief Pain Inventory-Short Form questionnaires). Toxicity was assessed according to Common Terminology Criteria for Adverse Events (CTCAE).

Results indicated that the most common side effects were dry mouth, fatigue and nausea, none of which exceeded grade 2 in severity. In terms of the outcomes, an objective response in nodal or visceral disease was reported in $82 \%$ of patients with measurable disease, together with clinically meaningful improvements in pain severity and quality of life, as early as after the second cycle of therapy. The authors concluded that radionuclide treatment with ${ }^{177} \mathrm{Lu}$-PSMA-617 has a high treatment-response rate with low toxic effects, and reduction of pain in men with metastatic castration-resistant prostate cancer who have progressed after conventional treatments (24).

Emmet et al. (31) from the St Vincent's Hospital in Sydney, Australia, conducted a prospective phase 2 study which focused on imaging predictors of treatment response and patterns of disease progression. Eligibility criteria included uptake on PSMA PET above or equal to liver activity, in the absence of any ${ }^{18} \mathrm{~F}-\mathrm{FDG}$ PETdiscordant disease. Study participants received up to 4 cycles of ${ }^{177} \mathrm{Lu}$-PSMA at 6 weekly intervals, after which imaging was repeated. Treatment response to ${ }^{177} \mathrm{Lu}$-PSMA was assessed using PSA response and comparison to molecular imaging parameters at baseline. The authors concluded that "PSMA PET plays an important role in predicting treatment response to Lu-PSMA and in identifying subsequent patterns of failure, which may aid in determining the next best treatment options". Several retrospective studies have also suggested similar efficacy and toxicity profiles (31).

A recent meta-analysis and systematic review on RLT with ${ }^{177} \mathrm{LuPSMA}$ for metastatic castrate-resistant prostate cancer suggested that this is an effective treatment of advanced stages of the disease that is refractory to standard therapeutic options and that it has a low toxicity profile (20). In another review on the use of ${ }^{177} \mathrm{LuPSMA}$ in the setting of metastatic prostate cancer, the authors concluded that ${ }^{177} \mathrm{Lu}-\mathrm{PSMA}$ RLT is a safe and promising form of treatment especially in patients who have progressed beyond standard treatment. Considering its low toxicity, ${ }^{177} \mathrm{Lu}$-PSMA RLT is additionally an ideal therapeutic option for patients who do not tolerate docetaxel therapy well or those who have extensive bone marrow involvement. Also, when compared to established systemic therapies, ${ }^{177} \mathrm{Lu}$-PSMA RLT results in PSA reductions and lengthens overall survival and progression-free survival with low-grade, transient toxicity (32). 


\section{${ }^{225}$ AC-PSMA}

The most promising targeted alpha-emitters for RLT included Actinium-225 $\left({ }^{225} \mathrm{Ac}\right)$, Bi-213 ${ }^{\left({ }^{13} \mathrm{Bi}\right)}$ and Radium-223 $\left({ }^{223} \mathrm{Ra}\right)$. Although some initial promising evidence for the use of ${ }^{213} \mathrm{Bi}-\mathrm{PSMA}$ was presented (33), it has since been realized that its physical characteristics makes ${ }^{213} \mathrm{Bi}$ a less than ideal candidate. The use of ${ }^{225}$ Ac-PSMA has been most extensively evaluated in humans and seem to provide the best therapeutic possibilities of the three alpha emitters.

${ }^{225}$ Ac-PSMA is an alpha-emitter with a relatively long half-life of 9.9 days. Its decay scheme results in the emission of four alpha particles and two beta particles, which together with the long half-life make for a rather cytotoxic radioisotope (34). The therapeutic use of an alpha-emitter offers several advantages. Its high linear energy transfer together with the short radiation range in human tissue results in multiple double-stranded DNA breaks which are largely independent of the cell cycle and oxygenation state, whilst leaving the surrounding unaffected tissues relatively unscathed. It offers an alternative form of therapy when conventional therapies (such as chemotherapy or external radiation) have resulted in resistance. A potential additional advantage is more pronounced abscopal effect when compared to beta radiation in preclinical studies. This effect relates to systemic tumor regression outside of the treated areas and may lead to favorable combinations with immunotherapy, such as immune checkpoint inhibitors $(34,35)$. Combining alpha-emitters with low molecular weight ligands that are internalized lead to fast tumor uptake and non-target clearance with a more favorable red marrow toxicity profile. Targeted alpha therapies have been in use for decades and have been successfully used in the treatment of brain tumors, neuro-endocrine tumors, prostate cancer and others (34).

Targeted alpha therapy appears to be particularly well-suited for application in combination with other forms of conventional therapy for the ablation of micrometastases, in patients with diffuse bone marrow infiltration, and in patients who have become resistant to other conventional therapy (34). The practical aspects of ${ }^{225}$ Ac-PSMA with regards to patient selection, preparation, administration, and minimization of side effects has been described (36) but standardized international guidelines are still lacking.

\section{Dose determination, dosimetry, and administration}

Dose determination of ${ }^{225}$ Ac-PSMA was established by Kratochwil et al. (37), based on time-activity curves that made use of dosimetry estimates obtained from serial ${ }^{177} \mathrm{Lu}$-PSMA scans obtained post-therapy which were then extrapolated to the physical half-life of ${ }^{225} \mathrm{Ac}$. Fourteen patients were divided into four groups with different empirical doses for salvage therapy as follows: $50 \mathrm{kBq} / \mathrm{kg}(\mathrm{n}=4), 100$ $\mathrm{kBq} / \mathrm{kg}(\mathrm{n}=4), 150 \mathrm{kBq} / \mathrm{kg}(\mathrm{n}=2)$ and $200 \mathrm{kBq} / \mathrm{kg}(\mathrm{n}=4)$. Treatment response and the presence of any toxicity was retrospectively evaluated. Results indicated the highest radiation dose was received by the salivary glands (2.3Sv), followed by the kidneys $(0.7 \mathrm{~Sv})$ and the bone marrow $(0.05 \mathrm{~Sv})$. The researchers found that xerostomia became the dose-limiting factor at treatment activities above $100 \mathrm{kBq} / \mathrm{kg} /$ cycle. Therapy administered at a dose of $100 \mathrm{kBq} / \mathrm{kg}$ resulted in a significant decline in s-PSA, which had a duration of less than 4 months, which was then 
improved with further cycles administered every two months. Administered activities of $50 \mathrm{kBq} / \mathrm{kg}$ resulted in a poor tumor control with no occurrence of side effects. Based on the aforementioned data, $100 \mathrm{kBq} / \mathrm{kg} / \mathrm{cycle}$ was determined as the optimal dose for human use with intervals of eight weeks in-between doses (37). A recently published 'image of the month' demonstrated the feasibility of image-based dosimetry with ${ }^{225} \mathrm{Ac}$-PSMA quantitative SPECT (single photon emission computed tomography) (38). Micro dosimetry calculations have also been calculated with Monte Carlo simulations (39).

\section{Clinical evidence with ${ }^{225}$ Ac-PSMA}

Clinical evidence has been limited mostly to retrospective observational studies at this point, with the majority of treated patients having received ${ }^{225} \mathrm{Ac}-\mathrm{PSMA}$ in a salvage therapy setting. A paper by Kratochwil and colleagues (40) evaluated tumor control duration and efficacy in a group of 40 patients with metastatic castrate-resistant prostate cancer. A dose of $100 \mathrm{kBq} / \mathrm{kg}$ was administered at intervals of two months for three cycles, and six-month follow-up evaluation performed with PSMA-based PET or SPECT imaging. Interim evaluations consisted of s-PSA and full blood count results, done monthly.

Outcomes considered consisted of the duration of the s-PSA response as well as the progression-free response based on the six-month radiological response. Their results demonstrated that $87 \%$ of included patients $(n=38)$ demonstrated a decrease in s-PSA and that $63 \%$ of patients $(n=33)$ demonstrated a s-PSA response of greater than $50 \%$. Median duration of tumor control was nine months, with five patients even exceeding two-year survival. This is indeed a remarkable response considering the very late stage of disease (when all other treatment options had been exhausted) in this group of patients when compared to conventional therapies used earlier-on in the treatment landscape. Such treatments include abiraterone, demonstrating a median duration of tumor control of 10 months, and docetaxel of 6.5 months. Xerostomia was the main side effect and led to discontinuation of therapy in four patients. The authors commented on a perceived higher efficacy compared to ${ }^{177} \mathrm{Lu}$-PSMA with salivary gland toxicity as the major limiting factor. The surrogate markers s-PSA and radiological progressionfree survival seemed to correspond well to the duration of tumor control (40).

Sathekge et al. published a study based on 57 patients, started on doses of either 10MBq (in cases such as those with a high bone tumor burden) or $8 \mathrm{MBq}$ which was then de-escalated based on the remaining tumor burden and occurrence of side effects. Radiological measurements and s-PSA were used as surrogate markers and patients were divided into two groups based on previous therapies received. Group A consisted of patients who received combinations of conventional therapy such as surgery, radiation therapy and/or androgen deprivation therapy. A $71 \%$ decrease in tumor markers was found in group A, compared to a 92\% response rate in Group B that consisted of patients who received minimal or no previous therapy. The majority of these patients reported an improvement in Quality of Life (with decreased bone pain) and minimal side effects. Group B also included patients who were chemotherapy naïve, due to ineligibility or unwillingness to undergo chemotherapy, who demonstrated significantly higher response rates and even complete responses (41). 
Another publication on a larger patient cohort by the same group reports on possible outcome predictors in patients with metastatic castrate-resistant prostate cancer treated with ${ }^{225}$ Ac-PSMA. The study population comprised of 73 patients treated with 210 cycles of ${ }^{225}$ Ac-PSMA-617. Special investigations that were routinely performed included full blood count, kidney function including glomerular filtration rate, liver function tests, s-PSA and ${ }^{68}$ Ga-PSMA PET/CT. Eighty-three percent of these patients demonstrated a favorable s-PSA response, with greater than $70 \%$ demonstrating a decline in s-PSA exceeding 50\%. Indicators of a positive treatment response included the magnitude of the s-PSA decline, whereas patients that were previously treated with ${ }^{177}$ Lu-PSMA had a poorer prognosis. The results further demonstrated a complete treatment response in 29\% of participants and estimated the median progression-free survival and overall survival at 15 months and 18 months, respectively. Xerostomia was reported in the vast majority of patients (85\%) but was not severe enough to discontinue therapy. No Grade IV bone marrow toxicity was reported, and renal toxicity occurred only in a small number of patients with baseline renal impairment. From these results, it is clear that ${ }^{225}$ Ac-PSMA-617 is indeed a viable treatment option for patients who have castration-resistant prostate carcinoma and for whom conventional therapy has failed. Treatment response is durable, and the side effects are tolerable (42).

A more recent prospective study from India (43) evaluated response and outcomes in 28 patients recruited with metastatic castrate-resistant cancer. Participants included a mixture of patients who demonstrated resistance to ${ }^{177} \mathrm{Lu}-\mathrm{PSMA}(\mathrm{n}=15,[54 \%])$ and others who had not been previously treated with ${ }^{177} \mathrm{Lu}-\mathrm{PSMA}(\mathrm{n}=13$ [46\%]), the majority of whom had extensive skeletal metastases on baseline imaging. This study population also included a significant number of patients (72\%) with ECOG 3-4 unlike other study populations. A dose of $100 \mathrm{kBq} / \mathrm{kg}$ was administered at two-month intervals and responses were evaluated based on s-PSA according to the prostate cancer working group criteria (PCWG3). Other parameters that were evaluated included overall survival, progression-free survival, disease control rate, tumor response according to PERCIST 1 criteria and the occurrence of side effects based on the CTCAE v5 criteria. The authors reported a $>50 \%$ decline in s-PSA for 39\% of patients at the end of follow-up, with a median progression-free survival of 12 months and a median overall survival of 17 months. The authors hypothesized that the slightly inferior results, when compared to other groups, could be attributable to the high percentage of patients with high ECOGs (ECOG 3-4) that were included. Decreases in s-PSA was again identified as a good prognostic indicator and vice versa and side effects experienced were limited to Grade I/II toxicity. Sub-group analysis comparing the outcomes of patients with prior exposure to ${ }^{177} \mathrm{Lu}$-PSMA compared to those naive to ${ }^{177} \mathrm{Lu}$-PSMA demonstrated a greater than $50 \%$ decline in $53.8 \%$ of patients compared to only $26.6 \%$ in the previously exposed group. Similar to previous studies that demonstrated promising disease control rates with ${ }^{225}$ Ac-PSMA, this study also showed low toxicity (43). There are a number of impressive case studies that demonstrate remarkable treatment responses to ${ }^{225}$ Ac-PSMA in patients with significant visceral metastases, such as brain, lungs and orbital (44-46). The outcomes of several current large multi-center trials are eagerly anticipated. 


\section{COMPARING ${ }^{177}$ LU-PSMA AND ${ }^{225}$ AC-PSMA}

To the best of the authors' knowledge, no prospective head-to-head comparisons have been performed to establish which form of targeted radionuclide therapy is more effective. Theoretically, ${ }^{225} \mathrm{Ac}$-PSMA produces more double-stranded DNA breaks within the tumor cells, which should lead to a higher efficacy. It is also better suited to patients with significant skeletal metastases as the shorter tissue penetration should spare the bone marrow to a greater extent than ${ }^{177} \mathrm{Lu}-\mathrm{PSMA}$ with its $\beta$-particle emission. This unfortunately comes at the cost of significant toxicity to the salivary glands with a non-negligible effect on quality of life. Figure 2 suggests a possible pathway for treatment selection in patients with metastatic castration-resistant prostate cancer. Some centers prefer to treat patients with mainly soft tissue involvement with ${ }^{177} \mathrm{Lu}-\mathrm{PSMA}$ and to reserve those with extensive skeletal involvement (with or without soft tissue involvement) for therapy with ${ }^{225}$ Ac-PSMA. The choice would of course further be dictated by what is available, what is funded, and what the patients' preferences are.

\section{${ }^{223}$ Ra-dicloride}

An overview of targeted alpha therapy in the setting of prostate cancer would hardly be complete without mentioning the use of ${ }^{223} \mathrm{Ra}$ (Radium-223). ${ }^{223} \mathrm{Ra}$ is an alpha particle emitter with a physical half-life of 11.4 days which acts on metastatic bone lesions due to its similarities to calcium in that it complexes with hydroxyapatite at areas of increased bone turnover. The ALSYMPCA trial was a large Phase III international multi-center trial that compared the efficacy of ${ }^{223} \mathrm{Ra}$ dichloride to that of a placebo in the setting of metastatic castrate-resistant prostate cancer. Participants consisted of those with symptomatic and progressive disease with at least two skeletal metastases, but without any visceral metastases and with an ECOG up to 2. Results from this trial consisting of over 900 participants demonstrated an improvement in overall survival of 3.6 months when compared to placebo and was subsequently approved for use by the FDA (47). Side effects were mostly related to bone marrow with resultant anemia, lymphopenia and thrombocytopenia. Long-term effects on the emergence of bone marrow-related cancers are unknown considering the lag effect of nearly 20 years needed and there are a number of other concerns and criticisms around this trial. Importantly, the use of bone scintigraphy in combination with CT in the screening process of participants seems sub-optimal in light of better detection modalities such as ${ }^{68} \mathrm{Ga}$-PSMA PET (48). The other limitation is that treatment is limited to skeletal metastases only and that any visceral metastases (such as lung, liver and brain with major impact on prognosis) are left untreated. The EANM has recently published a procedure guideline on the use of ${ }^{223} \mathrm{Ra}$-dicloride in the treatment of skeletal metastases in prostate cancer patients (49).

\section{COMBINATION THERAPIES}

Some research groups have adopted a tandem approach making use of both ${ }^{177} \mathrm{Lu}-\mathrm{PSMA}$ and ${ }^{225} \mathrm{Ac}$-PSMA in an attempt to increase efficacy whilst minimizing 
possible toxicity. In such approach, researchers from the Saarland University, Saarbrücken, Germany, administered $5 \mathrm{MBq}$ of ${ }^{225} \mathrm{Ac}$-PSMA in combination with 7 GBq of ${ }^{177} \mathrm{Lu}$-PSMA-617. Results indicated a good s-PSA response after a single course of low activity ${ }^{225}$ AcPSMA combined with a full activity of ${ }^{177} \mathrm{Lu}$-PSMA with reduced salivary gland toxicity (50). Kulkarni et al. also used a similar approach making use of 2-7 MBq of ${ }^{225} \mathrm{Ac}-\mathrm{PSMA}$ in combination with 3-7.5 GBq of ${ }^{177}$ Lu-PSMA-617 (51).

To date, there have been no clinical trials evaluating the effects of ${ }^{225}$ AcPSMA in combination with chemotherapy. Often patients have had chemotherapy at the point when they are referred for targeted alpha therapy and good efficacy is still achieved. Currently, it seems that conventional therapies are complimentary to ${ }^{225}$ Ac-PSMA and vice versa. The best patient management will take place in the setting of a multi-disciplinary team, where treatment options can be introduced as needs arise. External beam radiation therapy is often needed post targeted alpha therapy in instances where one or two metastatic lesions remain which do not justify systemic therapy.

Targeted alpha therapy provides radiation with high linear energy transfer, whereby even a single alpha particle traversing a DNA strand results in doublestranded DNA breaks with blunt edges that are difficult to repair. Despite this effective mechanism, poor response or resistance is not negligible (despite sufficient PSMA expression) and is likely related to DNA repair pathways. A pilot study by Kratochwil et al. investigated 10 patients with poor responses to TAT despite sufficient PSMA expression with CT-guided biopsy and targeted nextgeneration sequencing. Histology could be obtained in seven lesions, which identified increases in the following mutations in DNA-damage recognition: ATM, CHEK-2 and TP53. The authors concluded that the frequency of DNA damage-recognition and signaling-checkpoint genes appeared increased in non-responders. Unfortunately, this study was performed in the absence of a control group, which makes the normal prevalence of these phenomena difficult to assess. This group also commented that the occurrence of gene mutations coding for BRCAl/2 tended to be rare (52). The implications from these findings are that the combination of TAT with PARP-inhibitors and/or immunotherapies may be beneficial under certain conditions, particularly in poor responders.

Future combinations with DNA damage-repair targeting agents such as poly (ADP-ribose)-polymerase inhibitors (PARPi), such as Olaparib, in patients with germline/ somatic mutations of especially ATM, BRCA1 and BRCA2 seems to be a reasonable approach, and this will be evaluated in ongoing prospective trials. Combination with immune checkpoint inhibitors also provide a potentially exciting therapeutic development (53). Figure 3 summarizes the potential mechanisms to optimize PSMA therapy based on a diagram by Kumar et al. (53).

\section{TREATMENT RESPONSE EVALUATION CRITERIA}

In the majority of published trials on targeted alpha therapy, treatment response evaluation is based on criteria similar to those used by the Prostate Cancer Clinical 


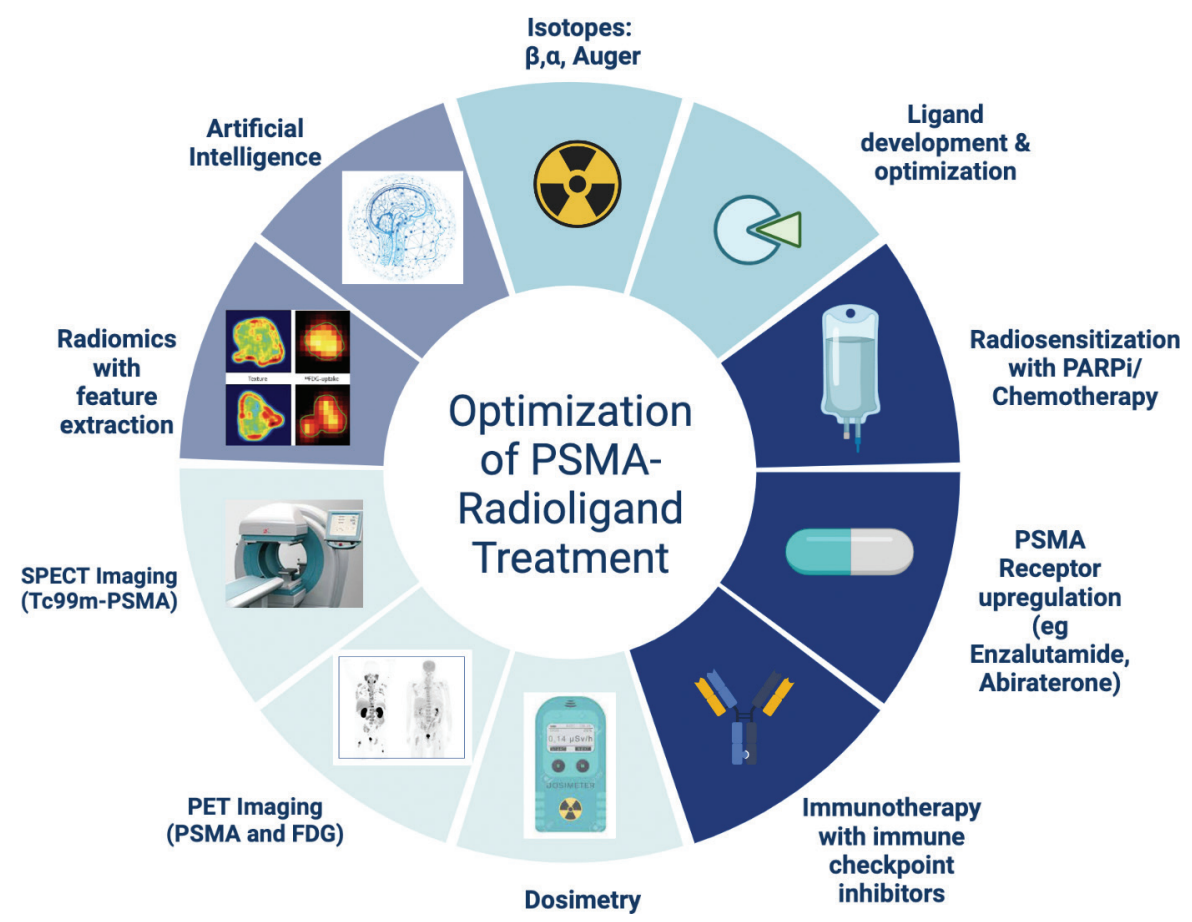

Figure 3. Optimization of prostate cancer treatment with PSMA radionuclide therapy. A combination of various strategies is needed to optimize selection and treatment for patients with metastatic CRPC. TAT combined with chemotherapy, immunotherapy and PARPinhibitors may increase the effectiveness of therapy over that of a single PSMA-based radionuclide agent, especially in those patients with non-congruent uptake on FDG PET. (Based on the diagram by Kumar et al Clinical Cancer Research. 2020; 26(12):2774-6.)

Trials Working Group 2/3 (54) which include clinical and laboratory findings together with conventional imaging modalities (CT, magnetic resonance imaging, and bone scan). Some groups have also included PSMA-based imaging although these have not yet been standardized by means of an international guideline. In a recent editorial published in the Journal of Nuclear Medicine, Fanti et al. (55) proposed the development of robust systemic treatment response assessment criteria at the time of PSMA imaging, which they coined "PSMA PET Progression Criteria (PPP)". They propose the following three criteria: (i) the appearance of at least two new lesions that are PSMA-positive and at distant sites; (ii) appearance of one new PSMA-positive lesion, which corresponds to clinical and laboratory data, and which is confirmed either by biopsy or with correlative imaging within three months of the PSMA PET; and (iii) an increase in size or intensity of PSMA uptake in at least one lesion by at least 30\% which corresponds to clinical and laboratory data and is confirmed with biopsy or correlative imaging within three months of the PET study (55). 


\section{CONCLUSION}

It is anticipated that further developments with regards to the type of PSMA and delivery thereof will continue in an effort to maximize the tumor radiation dose whilst limiting radiation to particularly the salivary glands. Improved dosimetry modeling may also assist in the individualization of doses with the need for doseescalation and de-escalation based on individual patient imaging. This would also apply to establishing ideal treatment intervals and the total number of cycles needed. There is a need for formalized international guidelines with regards to patient selection and treatment regimens as well as the interpretation and reporting of treatment response on PSMA-based imaging. It is further hoped that ${ }^{225}$ Ac-PSMA will be registered and produced worldwide based on emerging data from prospective randomized trials. It will earn its deserved spot in the treatment landscape of patients with metastatic castrate resistant prostate cancer. It is anticipated that earlier on in the treatment schedule, patients will receive greater benefits either as a single therapy, or in combination with various therapies that may include ${ }^{177} \mathrm{Lu}$-PSMA, chemotherapy, androgen-deprivation therapy and immunecheckpoint inhibitors.

Conflict of interest: The authors declare no potential conflicts of interest with respect to research, authorship and/or publication of this article.

Copyright and permission statement: The authors confirm that the materials included in this chapter do not violate copyright laws. Where relevant, appropriate permissions have been obtained from the original copyright holder(s), and all original sources have been appropriately acknowledged or referenced.

\section{REFERENCES}

1. Sridhar SS, Freedland SJ, Gleave ME, Higano C, Mulders P, Parker C, et al. Castration-Resistant Prostate Cancer: From New Pathophysiology to New Treatment. Eur Urol. 2014;65(2):289-99. https://doi.org/10.1016/j.eururo.2013.08.008

2. Babb C, Urban M, Kielkowski D, Kellett P. Prostate Cancer in South Africa: Pathology Based National Cancer Registry Data (1986-2006) and Mortality Rates (1997-2009). Prostate Cancer. 2014;1-9. https://doi.org/10.1155/2014/419801

3. Hotte SJ, Saad F. Current management of castrate-resistant prostate cancer. Curr Oncol. 17(Suppl 2): 72-9. https://doi.org/10.3747/co.v17i0.718

4. Gillessen S, Attard G, Beer TM, Beltran H, Bjartell A, Bossi A, et al. Management of Patients with Advanced Prostate Cancer: Report of the Advanced Prostate Cancer Consensus Conference 2019. Eur Urol. 2020;77(4):508-47. https://doi.org/10.1016/j.eururo.2020.01.012

5. Abdel-Rahman O. Efficacy and toxicity outcomes of elderly castrate-resistant prostate cancer patients treated with docetaxel-A pooled analysis of 3 randomized studies. Urol Oncol-Semin Ori. 2020;38(4):210-5. https://doi.org/10.1016/j.urolonc.2019.09.004

6. Yordanova A, Eppard E, Kürpig S, Bundschuh RA, Schönberger S, Gonzalez-Carmona M, et al. Theranostics in nuclear medicine practice. Oncotargets Ther. 2017;10:4821-8. https://doi.org/10.2147/ OTT.S140671

7. Drude N, Tienken L, Mottaghy FM. Theranostic and nanotheranostic probes in nuclear medicine. Methods. 2017;130:14-22. https://doi.org/10.1016/j.ymeth.2017.07.004 
8. Farolfi A, Lima GM, Oyen W, Fanti S. Molecular Imaging and Theranostics-A Multidisciplinary Approach. Semin Nucl Med. 2019;49(4):247-54. https://doi.org/10.1053/j.semnuclmed.2019.02.002

9. Perera M, Papa N, Roberts M, Williams M, Udovicich C, Vela I, et al. Gallium-68 Prostate-specific Membrane Antigen Positron Emission Tomography in Advanced Prostate Cancer-Updated Diagnostic Utility, Sensitivity, Specificity, and Distribution of Prostate-specific Membrane Antigen-avid Lesions: A Systematic Review and Meta-analysis. Eur Urol. 2019;77(4):403-17. https://doi.org/10.1016/j. eururo.2019.01.049

10. Kimura S, Abufaraj M, Janisch F, Iwata T, Parizi MK, Foerster B, et al. Performance of [68Ga] Ga-PSMA 11 PET for detecting prostate cancer in the lymph nodes before salvage lymph node dissection: a systematic review and meta-analysis. Prostate Cancer Prostatic Dis. 2020;23(1):1-10. https://doi. org/10.1038/s41391-019-0156-z

11. Tan N, Bavadian N, Calais J, Oyoyo U, Kim J, Turkbey IB, et al. Imaging of Prostate Specific Membrane Antigen Targeted Radiotracers for the Detection of Prostate Cancer Biochemical Recurrence after Definitive Therapy: A Systematic Review and Meta-Analysis. J Urol. 2019;202(2):231-40. https://doi. org/10.1097/JU.0000000000000198

12. Mason BR, Eastham JA, Davis BJ, Mynderse LA, Pugh TJ, Lee RJ, et al. Current Status of MRI and PET in the NCCN Guidelines for Prostate Cancer. J Natl Compr Canc Netw. 2019;17(5):506-13. https:// doi.org/10.6004/jnccn.2019.7306

13. Jadvar H. Is There Use for FDG-PET in Prostate Cancer? Semin Nucl Med. 2016;46(6):502-6. https:// doi.org/10.1053/j.semnuclmed.2016.07.004

14. Wang B, Liu C, Wei Y, Meng J, Zhang Y, Gan H, et al. A Prospective Trial of 68Ga-PSMA and 18F-FDG PET/CT in Nonmetastatic Prostate Cancer Patients with an Early PSA Progression During Castration. Clin Cancer Res. 2020;26(17):4551-8. https://doi.org/10.1158/1078-0432.CCR-20-0587

15. Bauckneht M, Capitanio S, Donegani MI, Zanardi E, Miceli A, Murialdo R, et al. Role of Baseline and Post-Therapy 18F-FDG PET in the Prognostic Stratification of Metastatic Castration-Resistant Prostate Cancer (mCRPC) Patients Treated with Radium-223. Cancers. 2019;12(1):31. https://doi. org/10.3390/cancers 12010031

16. Ranjan S. Lutetium-177 prostate-specific membrane antigen-617 theranostics: New therapeutic hope in metastatic castrate-resistant prostate cancer? Indian J Urol. 2020;36(3):227. https://doi. org/10.4103/iju.IJU_193_20

17. Fendler WP, Rahbar K, Herrmann K, Kratochwil C, Eiber M. 177Lu-PSMA radioligand therapy for prostate cancer. Chin J Nucl Medicine Mol Imaging. 2019;39(10):636-40.

18. Zang J, Fan X, Wang H, Liu Q, Wang J, Li H, et al. First-in-human study of 177Lu-EB-PSMA-617 in patients with metastatic castration-resistant prostate cancer. Eur J Nucl Med Mol I. 2019;46(1): 148-58. https://doi.org/10.1007/s00259-018-4096-y

19. Das T, Guleria M, Parab A, Kale C, Shah H, Sarma HD, et al. Clinical translation of 177Lu-labeled PSMA-617: Initial experience in prostate cancer patients. Nucl Med Biol. 2016;43(5):296-302. https://doi.org/10.1016/j.nucmedbio.2016.02.002

20. Delker A, Fendler WP, Kratochwil C, Brunegraf A, Gosewisch A, Gildehaus FJ, et al. Dosimetry for 177Lu-DKFZ-PSMA-617: a new radiopharmaceutical for the treatment of metastatic prostate cancer. Eur J Nucl Med Mol I. 2016;43(1):42-51. https://doi.org/10.1007/s00259-015-3174-7

21. Jones W, Griffiths K, Barata PC, Paller CJ. PSMA Theranostics: Review of the Current Status of PSMATargeted Imaging and Radioligand Therapy. Cancers. 2020;12(6):1367. https://doi.org/10.3390/ cancers 12061367

22. Emmett L, Willowson K, Violet J, Shin J, Blanksby A, Lee J. Lutetium 177 PSMA radionuclide therapy for men with prostate cancer: a review of the current literature and discussion of practical aspects of therapy. J Medical Radiat Sci. 2017;64(1):52-60. https://doi.org/10.1002/jmrs.227

23. Hofman MS, Hicks RJ, Maurer T, Eiber M. Prostate-specific Membrane Antigen PET: Clinical Utility in Prostate Cancer, Normal Patterns, Pearls, and Pitfalls. Radiographics. 2017;38(1):200-17. https:// doi.org/10.1148/rg.2018170108

24. Hofman MS, Violet J, Hicks RJ, Ferdinandus J, Thang SP, Akhurst T, et al. [177Lu]-PSMA-617 radionuclide treatment in patients with metastatic castration-resistant prostate cancer (LuPSMA trial): a single-centre, single-arm, phase 2 study. Lancet Oncol. 2018;19(6):825-33. https://doi.org/10.1016/ S1470-2045(18)30198-0 
25. Kratochwil C, Fendler WP, Eiber M, Baum R, Bozkurt MF, Czernin J, et al. EANM procedure guidelines for radionuclide therapy with 177Lu-labelled PSMA-ligands (177Lu-PSMA-RLT). Eur J Nucl Med Mol I. 2019;46(12):2536-44. https://doi.org/10.1007/s00259-019-04485-3

26. Vorster M, Warwick J, Lawal I, Toit P du, Vangu M, Nyakale N, et al. South African guidelines for receptor radioligand therapy (RLT) with Lu-177-PSMA in prostate cancer. S Afr J Surg. 2020;57(4):45-51. https://doi.org/10.17159/2078-5151/2019/v57n4a3107

27. Yadav MP, Ballal S, Tripathi M, Damle NA, Sahoo RK, Seth A, et al.. 177 Lu-DKFZ-PSMA-617 therapy in metastatic castration resistant prostate cancer: safety, efficacy, and quality of life assessment. Eur J Nucl Med Mol Imaging. 2017 Jan;44(1):81-91. https://doi.org/10.1007/s00259-016-3481-7

28. Baum RP, Kulkarni HR, Schuchardt C, Singh A, Wirtz M, Wiessalla S, et al. 177Lu-Labeled Prostate-Specific Membrane Antigen Radioligand Therapy of Metastatic Castration-Resistant Prostate Cancer: Safety and Efficacy. J Nucl Med. 2016;57(7):1006-13. https://doi.org/10.2967/ jnumed.115.168443

29. Bräuer A, Grubert LS, Roll W, Schrader AJ, Schäfers M, Bögemann M, et al. 177Lu-PSMA-617 radioligand therapy and outcome in patients with metastasized castration-resistant prostate cancer. Eur J Nucl Med Mol I. 2017;44(10):1663-70. https://doi.org/10.1007/s00259-017-3751-z

30. Gadot M, Davidson T, Aharon M, Atenafu EG, Malki A, Levartovsky M, et al. Clinical Variables Associated with PSA Response to Lutetium-177-PSMA ([177Lu]-PSMA-617) Radionuclide Treatment in Men with Metastatic Castration-Resistant Prostate Cancer. Cancers. 2020;12(5):1078. https://doi. org/10.3390/cancers 12051078

31. Emmett L, Crumbaker M, Ho B, Willowson K, Eu P, Ratnayake L, et al. Results of a Prospective Phase 2 Pilot Trial of 177Lu-PSMA-617 Therapy for Metastatic Castration-Resistant Prostate Cancer Including Imaging Predictors of Treatment Response and Patterns of Progression. Clin Genitourin Canc. 2019;17(1):15-22. https://doi.org/10.1016/j.clgc.2018.09.014

32. Kim YJ, Kim Y. Therapeutic Responses and Survival Effects of 177Lu-PSMA-617 Radioligand Therapy in Metastatic Castrate-Resistant Prostate Cancer. Clin Nucl Med. 2018;43(10):728-34. https://doi. org/10.1097/RLU.0000000000002210

33. Sathekge M, Knoesen O, Meckel M, Modiselle M, Vorster M, Marx S. 213Bi-PSMA-617 targeted alpha-radionuclide therapy in metastatic castration-resistant prostate cancer. Eur J Nucl Med Mol. 2017;44(6):1099-100. https://doi.org/10.1007/s00259-017-3657-9

34. Morgenstern A, Apostolidis C, Kratochwil C, Sathekge M, Krolicki L, Bruchertseifer F. An Overview of Targeted Alpha Therapy with 225 Actinium and 213 Bismuth. Curr Radiopharm. 2018;11(3):200-8. https://doi.org/10.2174/1874471011666180502104524

35. McDevitt MR, Ma D, Lai LT, Simon J, Borchardt P, Frank RK, et al. Tumor Therapy with Targeted Atomic Nanogenerators. Science. 2001;294(5546):1537-40. https://doi.org/10.1126/ science. 1064126

36. Ahmadzadehfar H, Rahbar K, Essler M, Biersack HJ. PSMA-Based Theranostics: A Step-by-Step Practical Approach to Diagnosis and Therapy for mCRPC Patients. Semin Nucl Med. 2019;50(1): 98-109. https://doi.org/10.1053/j.semnuclmed.2019.07.003

37. Kratochwil C, Bruchertseifer F, Rathke H, Bronzel M, Apostolidis C, Weichert W, et al. Targeted $\alpha$-Therapy of Metastatic Castration-Resistant Prostate Cancer with 225 Ac-PSMA-617: Dosimetry Estimate and Empiric Dose Finding. J Nucl Med. 2017;58(10):1624-31. https://doi.org/10.2967/ jnumed.117.191395

38. Gosewisch A, Schleske M, Gildehaus FJ, Berg I, Kaiser L, Brosch J, et al. Image-based dosimetry for 225Ac-PSMA-I\&T therapy using quantitative SPECT. Eur J Nucl Med Mol. 2020;1-2. https://doi. org/10.1007/s00259-020-05024-1

39. Gabiña PM, Roeske JC, Mínguez R, Rodeño E, Iturriaga AG de. Microdosimetry-based determination of tumour control probability curves for treatments with 225 Ac-PSMA of metastatic castration resistant prostate cancer. Phys Med Biol. 2020;65(23):235012. https://doi.org/10.1088/1361-6560/ abbc81

40. Kratochwil C, Bruchertseifer F, Rathke H, Hohenfellner M, Giesel FL, Haberkorn U, et al. Targeted $\alpha$-Therapy of Metastatic Castration-Resistant Prostate Cancer with 225Ac-PSMA-617: Swimmer-Plot Analysis Suggests Efficacy Regarding Duration of Tumor Control. J Nucl Med. 2018;59(5):795-802. https://doi.org/10.2967/jnumed.117.203539 
41. Sathekge M, Bruchertseifer F, Knoesen O, Reyneke F, Lawal I, Lengana T, et al. 225Ac-PSMA-617 in chemotherapy-naive patients with advanced prostate cancer: a pilot study. Eur J Nucl Med Mol I. 2019;46(1):129-38. https://doi.org/10.1007/s00259-018-4167-0

42. Sathekge M, Bruchertseifer F, Vorster M, Lawal I, Knoesen O, Mahapane J, et al. Predictors of overall and disease free survival in metastatic castration-resistant prostate cancer patients receiving 225Ac-PSMA-617 radioligand therapy. J Nucl Med. 2019;61(1):jnumed.119.229229. https://doi. org/10.2967/jnumed.119.229229

43. Yadav MP, Ballal S, Sahoo RK, Tripathi M, Seth A, Bal C. Efficacy and safety of 225Ac-PSMA-617 targeted alpha therapy in metastatic castration-resistant Prostate Cancer patients. Theranostics. 2020;10(20):9364-77. https://doi.org/10.7150/thno.48107

44. Sathekge MM, Bruchertseifer F, Lawal IO, Vorster M, Knoesen O, Lengana T, et al. Treatment of brain metastases of castration-resistant prostate cancer with 225Ac-PSMA-617. Eur J Nucl Med Mol I. 2019;46(8):1756-7. https://doi.org/10.1007/s00259-019-04354-z

45. Maserumule LC, Mokoala KMG, Hlongwa KN, Ndlovu H, Reed JD, Ismail A, et al. Exceptional initial response of prostate cancer lung metastases to 225Ac-PSMA: A case report. CPCCR. 2021;3:100038. https://doi.org/10.1016/j.cpccr.2020.100038

46. Parihar AS, Chandekar KR, Singh H, Sood A, Mittal BR. Orbital and brain metastases on 68Ga-PSMA PET/CT in a patient with prostate carcinoma refractory to 177Lu-PSMA and 225Ac-PSMA therapy. Asia Ocean J Nucl Med Biol. 2021;9(1):67-70.

47. Heidegger I, Pichler R, Heidenreich A, Horninger W, Pircher A. Radium-223 for metastatic castrationresistant prostate cancer: results and remaining open issues after the ALSYMPCA trial. Transl Androl Urol. 2018;7(Suppl 1):S132-4. https://doi.org/10.21037/tau.2017.10.06

48. Sartor O, Coleman RE, Nilsson S, Heinrich D, Helle SI, O'Sullivan JM, et al. An exploratory analysis of alkaline phosphatase, lactate dehydrogenase, and prostate-specific antigen dynamics in the phase 3 ALSYMPCA trial with radium-223. Ann Oncol. 2017;28(5):1090-7. https://doi.org/10.1093/annond $\operatorname{mdx} 044$

49. Poeppel TD, Handkiewicz-Junak D, Andreeff M, Becherer A, Bockisch A, Fricke E, et al. EANM guideline for radionuclide therapy with radium-223 of metastatic castration-resistant prostate cancer. Eur J Nucl Med Mol Imaging. 2018;45(5):824-45. https://doi.org/10.1007/s00259-017-3900-4

50. Khreish F, Ebert N, Ries M, Maus S, Rosar F, Bohnenberger H, et al. 225Ac-PSMA-617/177LuPSMA-617 tandem therapy of metastatic castration-resistant prostate cancer: pilot experience. Eur J Nucl Med Mol Imaging. 2020;47(3):721-8. https://doi.org/10.1007/s00259-019-04612-0

51. Kulkarni HR, Singh A, Schuchardt C, Niepsch K, Sayeg M, Leshch Y, et al. PSMA-Based Radioligand Therapy for Metastatic Castration-Resistant Prostate Cancer: The Bad Berka Experience Since 2013. J Nucl Med. 2016;57(Supplement_3):97S-104S. https://doi.org/10.2967/jnumed.115.170167

52. Kratochwil C, Giesel FL, Heussel C-P, Kazdal D, Endris V, Nientiedt C, et al. Patients Resistant Against PSMA-Targeting $\alpha$-Radiation Therapy Often Harbor Mutations in DNA Damage-Repair-Associated Genes. J Nucl Med. 2020;61(5):683-8. https://doi.org/10.2967/jnumed.119.234559

53. Kumar ASR, Hofman MS. Mechanistic Insights for Optimizing PSMA Radioligand Therapy. Clin Cancer Res. 2020;26(12):2774-6. https://doi.org/10.1158/1078-0432.CCR-20-0209

54. Scher HI, Morris MJ, Stadler WM, Higano C, Basch E, Fizazi K, et al. Trial Design and Objectives for Castration-Resistant Prostate Cancer: Updated Recommendations From the Prostate Cancer Clinical Trials Working Group 3. J Clin Oncol. 2016;34(12):1402-18. https://doi.org/10.1200/ JCO.2015.64.2702

55. Fanti S, Hadaschik B, Herrmann K. Proposal of Systemic Therapy Response Assessment Criteria in time of PSMA PET/CT imaging: PSMA PET Progression (PPP). J Nucl Med. 2019;61(5):678-82. https://doi.org/10.2967/jnumed.119.233817 